\title{
干燥方式对高氯酸铵/石墨烯复合材料的结构和热分解行为的影响
}

\author{
王学宝 ${ }^{1}$ 李晋庆 ${ }^{1,2}$ 罗运军 ${ }^{1, *}$ \\ ('北京理工大学材料学院, 北京 100081 ; 2 中国兵器科学研究院, 北京 100089)
}

\begin{abstract}
摘要: 通过溶胶-凝胶法制备了石墨烯水凝胶, 并将其与高氯酸铵(AP)复合, 然后分别采用自然干燥、冷冻干燥 和超临界 $\mathrm{CO}_{2}$ 干燥三种干燥方式制备了 AP/石墨烯复合材料, 并通过扫描电镜(SEM)、元素分析(EA)、X射线衍 射(XRD)、差示扫描量热仪(DSC)和热重-红外联用技术(TG-FTIR)研究了不同干燥方式对其结构和热分解行为 的影响. 结果表明, 干燥方式对 AP/石墨烯复合材料的形貌具有明显影响, 其中通过超临界 $\mathrm{CO}_{2}$ 干燥制备的 AP/ 石墨烯复合材料基本能保持与石墨烯气凝胶相似的外观和多孔结构. 通过自然干燥、冷冻干燥和超临界 $\mathrm{CO}_{2}$ 干 燥制备的 AP/石墨烯复合材料中 AP 的质量分数分别为 $89.97 \% 、 92.41 \%$ 和 $94.40 \%$, 其中通过超临界 $\mathrm{CO}_{2}$ 干燥 制备的复合材料中 AP 的粒径尺寸为 $69 \mathrm{~nm}$. DSC 测试结果表明, 石墨烯对 AP 的热分解过程具有明显的促进 作用, 能使 AP 的低温分解过程大大减弱, 高温分解峰温明显降低. 三种干燥方式相比, 通过超临界 $\mathrm{CO}_{2}$ 干燥制 备的 AP/石墨烯复合材料中石墨烯的促进作用最明显. 与纯 AP 相比, 其高温分解峰温降低了 $83.7^{\circ} \mathrm{C}$, 表观分 解热提高到 $2110 \mathrm{~J} \cdot \mathrm{g}^{-1}$. TG-FTIR 分析结果表明, AP/石墨烯复合材料的热分解过程中, AP 分解产生的氧化性 产物与石墨烯发生了氧化反应, 生成了 $\mathrm{CO}_{2}$.
\end{abstract}

关键词：干燥方式；石墨烯；高氯酸铵；溶胶-凝胶法；热分解

中图分类号: 0643

\section{Effect of Drying Methods on the Structure and Thermal Decomposition Behavior of Ammonium Perchlorate/Graphene Composites}

\author{
WANG Xue-Bao ${ }^{1} \quad$ LI Jin-Qing ${ }^{1,2} \quad$ LUO Yun-Jun ${ }^{1, *}$ \\ ('School of Material Science \& Engineering, Beijing Institute of Technology, Beijing 100081, P. R. China; \\ ${ }^{2}$ Academy of Ordnance Science, Beijing 100089, P. R. China)
}

\begin{abstract}
Graphene hydrogels were prepared by the sol-gel method, and then used to prepare ammonium perchlorate (AP)/graphene composites by the incorporation of AP. The composites were dried naturally in air, freeze-dried, or dried with supercritical $\mathrm{CO}_{2}$. Scanning electron microscopy (SEM), elemental analyses (EA), and X-ray diffraction (XRD) were used to characterize the structure of the AP/ graphene composites dried using different methods. Furthermore, the thermal decomposition behavior of the AP/graphene composites was investigated by differential scanning calorimetry (DSC) and thermogravimetric analysis/infrared spectroscopy (TG-FTIR). Drying method had an obvious influence on the morphology of the AP/graphene composites; only the AP/graphene composites dried with supercritical $\mathrm{CO}_{2}$ showed similar three-dimensional networks and porous structure to graphene aerogels. Elemental analyses revealed that the AP contents in the AP/graphene composites prepared by drying naturally, freeze-drying, and supercritical $\mathrm{CO}_{2}$ drying were $89.97 \%, 92.41 \%$, and $94.40 \%$, respectively. XRD results showed that AP was dispersed homogeneously on the nanoscale in the AP/graphene composites dried with supercritical $\mathrm{CO}_{2}$ and the average particle diameter of AP was about $69 \mathrm{~nm}$. DSC and TG-FTIR analyses indicated that graphene could promote the thermal decomposition of AP, particularly for the
\end{abstract}

Received: March 4, 2013; Revised: May 2, 2013; Published on Web: May 2, 2013.

"Corresponding author. Email: yjluo@bit.edu.cn; Tel: +86-10-68913698.

The project was supported by the Basic Research Foundation of Beijing Institute of Technology, China (20110942019).

北京理工大学基础研究基金(20110942019)资助项目

(C) Editorial office of Acta Physico-Chimica Sinica 
sample dried with supercritical $\mathrm{CO}_{2}$. Independent of drying method, the low-temperature decomposition of the as-prepared AP/graphene composites was not observed and the high-temperature decomposition was accelerated. Compared to the other two drying methods, graphene in the AP/graphene composites dried with supercritical $\mathrm{CO}_{2}$ showed most obvious promoting effects. The high-temperature decomposition temperature of the $\mathrm{AP} / \mathrm{graphene}$ composites dried with supercritical $\mathrm{CO}_{2}$ decreased by $83.7{ }^{\circ} \mathrm{C}$ compared with that of pure AP, and the total heat release reached $2110 \mathrm{~J} \cdot \mathrm{g}^{-1}$. Moreover, graphene also took part in the oxidation reactions with oxidizing products, which was confirmed by the generation of $\mathrm{CO}_{2}$.

Key Words: Drying method; Graphene; Ammonium perchlorate; Sol-gel method; Thermal decomposition

\section{1 引言}

高氯酸铵(AP)是复合固体火箭推进剂的重要氧 化剂, 其燃烧和热分解性能与推进剂的燃烧性能密 切相关. ${ }^{1,2}$ 通过添加少量添加剂可以有效地调节 AP 的分解温度, 显著增加 AP 的表观分解热. ${ }^{3-8}$ 在众多 添加剂中, 诸如炭黑、富勒烯和碳纳米管等碳材料 因具有独特的结构和优异的综合性能, 在对 AP 的 热分解过程中表现出良好的促进作用, ${ }^{9,10}$ 因而引起 了人们的关注.

石墨烯是由单层碳原子组成的具有二维蜂窝 状结构的新型碳材料, ${ }^{11}$ 是构建其他维度碳材料的 基本结构单元. ${ }^{12}$ 石墨烯具有超大的理论比表面积、 优异的电学、热学和力学等性能, ${ }^{13-16}$ 近几年成为全 世界范围内的一个研究热点. 石墨烯干凝胶和石墨 烯气凝胶等以石墨烯为基本结构单元的三维结构 石墨烯材料是具有低密度、高比表面积和高度多孔 性的纳米材料, ${ }^{17-19}$ 它们的制备一般是首先通过溶 胶-凝胶法制备出石墨烯湿凝胶, 然后经过冷冻干燥 或者超临界 $\mathrm{CO}_{2}$ 干燥制得. 由于石墨烯的优异性能 以及三维结构石墨烯材料的特殊结构, 使得三维结 构石墨烯材料成为制备复合材料的理想载体. 因 此, 将 AP 填充到三维结构石墨烯材料的孔隙内, 制 备出 $\mathrm{AP} /$ 石墨烯复合材料成为了可能. 以这种方式 实现 AP 与石墨烯的复合, 可以有效减小 AP 的粒 径, 确保石墨烯与 AP 的充分接触, 从而充分发挥石 墨烯对 AP 热分解的促进作用.

本文以氧化石墨烯为前驱体, 通过溶胶-凝胶法 制备了石墨烯水凝胶, 并通过溶液渗透过程将其与 AP 复合, 然后分别采用自然干燥、冷冻干燥和超临 界 $\mathrm{CO}_{2}$ 干燥三种干燥方式制备了 $\mathrm{AP} /$ 石墨烯复合材 料, 并用扫描电镜 $(\mathrm{SEM})$ 、元素分析(EA)、X射线衍 射(XRD)等对其结构进行了表征, 同时研究了不同 干燥方式对所制备的 AP/石墨烯复合材料的热分解
行为的影响.

\section{2 实验部分}

\section{1 主要原料}

石墨: 分析纯, 粒径 $\leq 48 \mu \mathrm{m}$, 国药集团化学试剂 有限公司; 硫酸、盐酸、过硫酸钾、五氧化二磷、高锰 酸钾、抗坏血酸和乙酸乙酯: 分析纯, 北京化工厂; 高氯酸铵: 工业级, 大连北方氯酸钾有限公司.

\section{$2.2 \mathrm{AP} /$ 石墨烯复合材料的制备}

氧化石墨用改进的 Hummers 法 ${ }^{20}$ 制备. 将一定 质量的氧化石墨加入到去离子水中, 搅拌后形成氧 化石墨的悬浊液, 然后超声波分散 1.5-2.0 h, 可得 到氧化石墨烯的水溶液. 在 $250 \mathrm{~mL}$ 的烧杯中加入 $50 \mathrm{~mL}$ 氧化石墨烯的水溶液(浓度为 $4 \mathrm{mg} \cdot \mathrm{mL}^{-1}$ ), 然 后加入 $1 \mathrm{~g}$ 抗坏血酸, 搅拌均匀后, 在 $40^{\circ} \mathrm{C}$ 下静置 $16 \mathrm{~h}$ 后形成石墨烯水凝胶. 将石墨烯水凝胶用去离 子水置换一周时间，以除去其中所含的过量抗坏血 酸及其氧化产物. 然后将石墨烯水凝胶放入 $\mathrm{AP}$ 的 饱和溶液中, 在 $85{ }^{\circ} \mathrm{C}$ 下浸泡 $5 \mathrm{~d}$ 后, 分别采用三种 干燥方式获得 AP/石墨烯复合材料: (1) 自然干燥; (2) 冷冻干燥; (3) 将含有饱和 $\mathrm{AP}$ 水溶液的石墨烯水 凝胶用乙酸乙酯置换 $3 \mathrm{~d}$, 再通过超临界干燥 $\mathrm{CO}_{2}$ 干 燥.

作为对比, 还将 $\mathrm{AP}$ 与石墨烯(以抗坏血酸为还 原剂制备)按比例混合, 通过研磨法制备了三种 AP 与石墨烯的机械共混物. 三种 AP 与石墨烯的机械 共混物中 $\mathrm{AP}$ 的质量分数分别与通过自然干燥、冷 冻干燥和超临界 $\mathrm{CO}_{2}$ 干燥制备的 $\mathrm{AP} /$ 石墨烯复合材 料中 $\mathrm{AP}$ 的质量分数相同.

\section{3 结构与性能表征}

超临界流体萃取装置, 美国 Thar 公司 SFE1000 型; 场发射扫描电子显微镜, 日本 HITACHI S-4800 型, 样品经喷金后观察; 元素分析仪, 德国 Elementar 
公司 Vario MICRO CUBE 型, 分别从块状样品内部 上、中、下三处具有代表性的部位取样, 分析结果的 平均值作为样品的最终分析结果; $\mathrm{X}$ 射线衍射, 荷兰 PANalytical 公司 X'Pert Pro MPD 型, $\mathrm{Cu} K_{\alpha}$ 靶 ( $\lambda=$ $0.15406 \mathrm{~nm})$, 测试电压和电流分别为 $40 \mathrm{kV}$ 和 40 $\mathrm{mA}$, 将样品研磨为粉末状后测试; TG-DSC 热分析 仪, 瑞士 METTLER TOLEDO TGA/DSC1 型, 加热 范围 30-600 ${ }^{\circ} \mathrm{C}$, 升温速率 $10{ }^{\circ} \mathrm{C} \cdot \mathrm{min}^{-1}$, 样品装在 $\mathrm{Al}_{2} \mathrm{O}_{3}$ 坩埚里, $\mathrm{Ar}$ 气氛, 流速 $20 \mathrm{~mL} \cdot \mathrm{min}^{-1}$; 与其联用 的 FTIR 光谱仪为美国 Thermo Scientific Nicolet iS10 红外光谱仪, 联通加热管温度为 $200{ }^{\circ} \mathrm{C}$, 红外 光谱仪气体池温度为 $210^{\circ} \mathrm{C}$.

\section{3 结果与讨论}

\section{1 干燥方式对 AP/石墨烯复合材料形貌的影响}

图 1 分别是通过不同干燥方式制备的 $\mathrm{AP} /$ 石墨 烯复合材料的形貌和 SEM 图. 从图 1(a)可以看到, 通过自然干燥制备的 $\mathrm{AP} /$ 石墨烯复合材料体积收缩 严重, 且其表面析出了大量 AP. 如图 1(c)所示, 通过 冷冻干燥制备的 $\mathrm{AP} /$ 石墨烯复合材料结构非常疏 松, 并有少量 AP 在表面析出. 这主要是冷冻干燥过 程中, 水凝胶中的水形成冰晶, 体积膨胀, 对石墨烯 水凝胶骨架的交联结构造成了一定的破坏导致的. 如图 1(e)所示, 通过超临界 $\mathrm{CO}_{2}$ 干燥制备的 $\mathrm{AP} /$ 石墨 烯复合材料基本能保持与石墨烯气凝胶相似的外
观. ${ }^{17}$

图 1(b) 是通过自然干燥制备的 $\mathrm{AP} /$ 石墨烯复合 材料的 SEM 图, 可以看到, 由于发生严重收缩, 石墨 烯团聚在一起, 已经看不到孔结构的存在. 如图 1(d) 所示, 通过冷冻干燥制备的 AP/石墨烯复合材料中 多孔结构同样不明显, 石墨烯骨架坍塌, 堆积在一 起. 从图 1(f) 可以看到, 通过超临界 $\mathrm{CO}_{2}$ 干燥制备的 $\mathrm{AP} /$ 石墨烯复合材料具有与石墨烯气凝胶类似的多 孔结构, 但是与后者的不同之处在于其石墨烯骨架 上析出了大量的 AP.

\section{2 干燥方式对 AP/石墨烯复合材料中 AP 含量的 影响}

$\mathrm{AP} /$ 石墨烯复合材料中 AP 的含量可以通过元 素分析得到. 由于 $\mathrm{AP} /$ 石墨烯复合材料中只有 $\mathrm{AP}$ 含 有 $\mathrm{N}$ 元素, 通过元素分析对 $\mathrm{N}$ 元素含量进行测定, 可 以计算出 AP 的含量. 根据元素分析结果计算出通 过自然干燥、冷冻干燥和超临界 $\mathrm{CO}_{2}$ 干燥制备的 $\mathrm{AP} /$ 石墨烯复合材料中 $\mathrm{AP}$ 的质量分数分别为 $89.97 \% 、 92.41 \%$ 和 $94.40 \%$, 这说明无论哪种干燥方 式制备的 $\mathrm{AP} /$ 石墨烯复合材内部均含有大量的 $\mathrm{AP}$, 并且不同干燥方式制备的 $\mathrm{AP} /$ 石墨烯复合材料中 $\mathrm{AP}$ 的含量略有差异. 不同干燥方式制备的 $\mathrm{AP} /$ 石墨 烯复合材料中 AP含量的不同主要与干燥过程有 关. 自然干燥过程中, 由于毛细管作用, AP 会随着石 墨烯水凝胶中的水迁移到凝胶表面，当水蒸发后 $\mathrm{AP}$
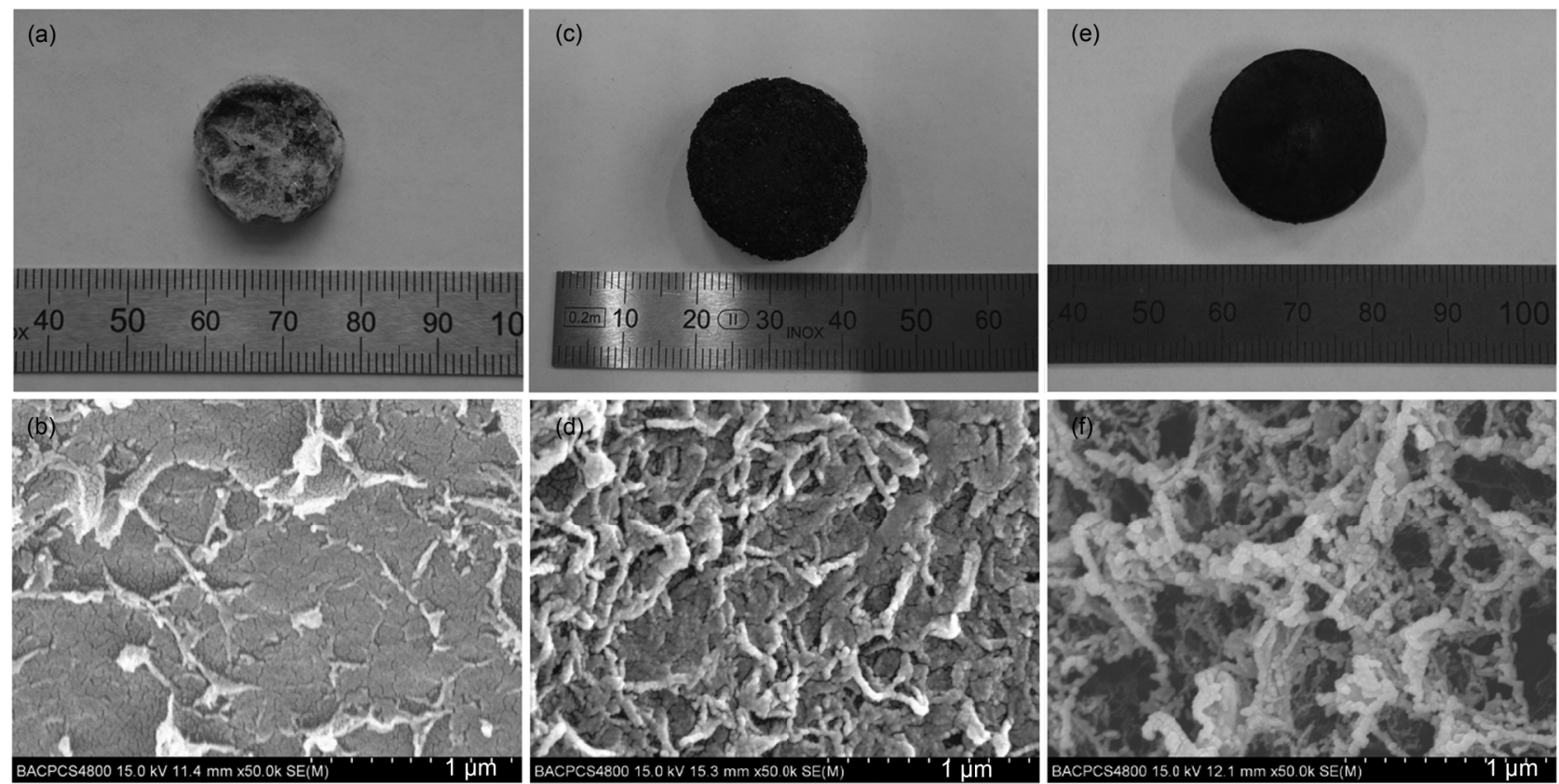

图 1 通过不同干燥方式制备的 AP/石墨烯复合材料的照片 $(a, c, e)$ 和 SEM 图 $(b, d, f)$

Fig.1 Photographs (a, c, e) and SEM images $(b, d, f)$ of the AP/graphene composites prepared by different drying methods $(a, b)$ drying naturally in atmosphere; (c, d) freeze drying; (e, f) supercritical $\mathrm{CO}_{2}$ drying 
留在了凝胶表面, 因而造成部分 AP 在复合材料表 面析出, 而其内部的 AP含量则减少. 冷冻干燥过程 中, 随着水气从升华界面逸出, 水气会夹带少量干 燥层中的 $\mathrm{AP}$ 微粒至石墨烯凝胶表面, 造成 $\mathrm{AP}$ 向石 墨烯凝胶表面的迁移，因而复合材料内部的 AP也 会减少. 超临界 $\mathrm{CO}_{2}$ 干燥之前, 要用乙酸乙酯置换石 墨烯水凝胶中的水, 由于 $\mathrm{AP}$ 在乙酸乙酯中的溶解 度很小, 这个过程中 AP 会在石墨烯凝胶内部析出, 并留在石墨烯凝胶内部. 与其他两种干燥方式相 比, 通过超临界 $\mathrm{CO}_{2}$ 干燥方式制备的 $\mathrm{AP} /$ 石墨烯复 合材料内部损失的 AP 相对较少.

\section{3 干燥方式对 AP/石墨烯复合材料中 AP 粒径的 影响}

图 2 是纯 $\mathrm{AP}$ 和通过不同干燥方式制备的 $\mathrm{AP} /$ 石墨烯复合材料的 XRD 衍射图. 纯 AP 和通过不同 干燥方式制备的 $\mathrm{AP} /$ 石墨烯复合材料都在相同的位 置出现明显的 AP 衍射峰, 说明通过不同干燥方式 制备的 $\mathrm{AP} /$ 石墨烯复合材料中 AP 的晶型没有发生 改变. 同时通过不同干燥方式制备的 $\mathrm{AP} /$ 石墨烯复 合材料的 XRD 衍射图中石墨烯的衍射峰均不明显, 这是因为复合材料中含有大量 AP, 石墨烯的含量相 对较少的缘故. 根据 Scherrer 公式, 通过自然干燥、 冷冻干燥和超临界 $\mathrm{CO}_{2}$ 干燥制备的 $\mathrm{AP} /$ 石墨烯复合 材料中 $\mathrm{AP}$ 的平均粒径分别为 $160 、 162$ 和 $69 \mathrm{~nm}$ (Scherrer 公式计算的晶粒尺寸范围在 1-100 nm 时 较为准确, 对于通过自然干燥和冷冻干燥制备的 $\mathrm{AP} /$ 石墨烯复合材料中 AP 的粒径尺寸, 此处仅粗略 表明其大于 $100 \mathrm{~nm})$, 说明以凝胶形式与石墨烯复 合后, $\mathrm{AP}$ 的粒径均大幅减小, 其中通过超临界 $\mathrm{CO}_{2}$

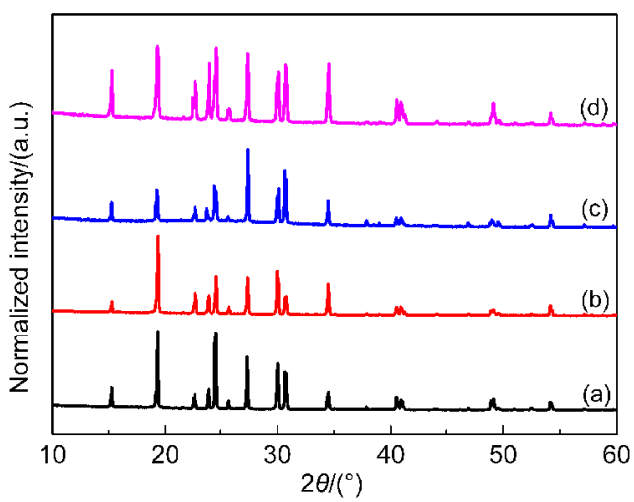

图 2 纯 $\mathrm{AP}(\mathrm{a})$ 、自然干燥 $(\mathrm{b})$ 、冷冻干燥(c)和超临界 $\mathrm{CO}_{2}$ 干燥(d)制备的 AP/石墨烯复合材料的 XRD 图

Fig.2 XRD patterns of pure AP (a) and AP/graphene composites prepared by drying naturally in atmosphere (b), freeze drying (c), and supercritical $\mathrm{CO}_{2}$ drying (d)
干燥制备的 $\mathrm{AP} /$ 石墨烯复合材料中 $\mathrm{AP}$ 的粒径为纳 米级.

\section{4 干燥方式对 AP/石墨烯复合材料热分解行为 的影响}

图 3 是纯 $\mathrm{AP}$ 、通过不同干燥方式制备的 $\mathrm{AP} /$ 石 墨烯复合材料和 AP与石墨烯的机械共混物的 DSC 曲线. 从图 3 可以看到, 在纯 AP 的 DSC 曲线上, $245.0^{\circ} \mathrm{C}$ 附近的吸热峰是 $\mathrm{AP}$ 的晶型转变吸热峰, 此 时 $\mathrm{AP}$ 由斜方晶型转变为立方晶型; $297.0^{\circ} \mathrm{C}$ 附近的 放热峰是 AP 的低温分解峰; $406.2{ }^{\circ} \mathrm{C}$ 附近的放热峰 是 AP 的高温分解峰. 高温分解是 AP 热分解的主要 分解过程, ${ }^{21}$ 此时 $\mathrm{AP}$ 完全分解. 从图 3 还可以看到, 通过不同干燥方式制备的 $\mathrm{AP} /$ 石墨烯复合材料和三 种 $\mathrm{AP}$ 与石墨烯的机械共混物的 DSC 曲线上, AP 的 晶型转变温度差别不大, 但是它们的热分解过程发 生了明显的变化. 与纯 $\mathrm{AP}$ 相比, 通过自然干燥制 备的 $\mathrm{AP} /$ 石墨烯复合材料的低温分解峰变得非常 微弱, 出现在 $293.3{ }^{\circ} \mathrm{C}$, 而高温分解峰则出现在 $336.5^{\circ} \mathrm{C}$, 降低了 $69.7^{\circ} \mathrm{C}$. 在冷冻干燥和超临界 $\mathrm{CO}_{2}$ 干燥制备的 $\mathrm{AP} /$ 石墨烯复合材料的 DSC 曲线上, 二 者的低温分解峰完全消失, 高温分解峰温分别降低 了 84.2 和 $83.7^{\circ} \mathrm{C}$. 而对于三种 $\mathrm{AP}$ 与石墨烯的机械 共混物来说, 三者的低温分解峰温比纯 AP均略有升 高, 高温分解峰温分别降低了 $36.0 、 42.1$ 和 $66.9^{\circ} \mathrm{C}$. 这说明石墨烯对 AP 的高温分解过程具有明显的促 进作用, 能促使 AP 的高温分解提前进行, 并且通过

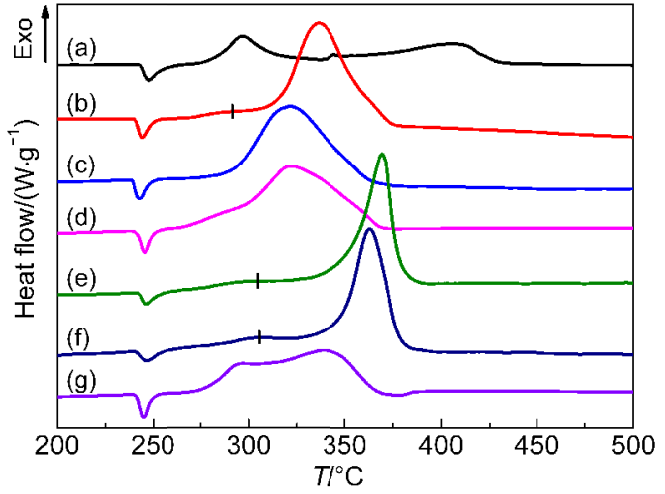

图 3 不同样品的 DSC 曲线

Fig.3 DSC curves of different samples

(a) pure $\mathrm{AP}$; (b) $\mathrm{AP} /$ graphene composites prepared by drying naturally in atmosphere; (c) $\mathrm{AP} /$ graphene composites prepared by freeze drying;

(d) $\mathrm{AP} /$ graphene composites prepared by supercritical $\mathrm{CO}_{2}$ drying;

(e) mechanical mixtures of AP with graphene ( $\left.w_{\mathrm{AP}}=89.97 \%\right)$;

(f) mechanical mixtures of AP with graphene ( $\left.w_{\mathrm{AP}}=92.41 \%\right)$; (g) mechanical mixtures of AP with graphene $\left(w_{\mathrm{AP}}=94.40 \%\right)$. $w_{\mathrm{AP}}$ is the mass fraction of AP in the mechanical mixtures of AP with graphene 
凝胶形式将石墨烯与 $\mathrm{AP}$ 复合比二者简单地机械共 混具有更明显的促进作用.

根据 $\mathrm{AP}$ 热分解的质子转移理论, ${ }^{21} \mathrm{AP}$ 的低温分 解是从质子由 $\mathrm{NH}_{4}^{+}$转移到 $\mathrm{ClO}_{4}^{-}$生成 $\mathrm{NH}_{3}$ 和 $\mathrm{HClO}_{4}$ 开 始的. $\mathrm{NH}_{3}$ 和 $\mathrm{HClO}_{4}$ 进入气相后，反应生成 $\mathrm{N}_{x} \mathrm{O}$ 、 $\mathrm{H}_{2} \mathrm{O} 、 \mathrm{O}_{2}$ 和 $\mathrm{HCl}$ 等产物. 由于低温反应阶段 $\mathrm{NH}_{3}$ 不能 被完全氧化, 未被氧化的 $\mathrm{NH}_{3}$ 会吸附到 $\mathrm{AP}$ 表面, 导 致低温分解结束. ${ }^{22}$ 随着温度的升高, $\mathrm{NH}_{3}$ 从 $\mathrm{AP}$ 表面 解吸附, 在气相中 $\mathrm{NH}_{3}$ 和 $\mathrm{HClO}_{4}$ 的氧化反应再次进 行, 此时 AP 的热分解进入高温分解阶段.

在 AP/石墨烯复合材料和 AP 与石墨烯的机械 共混物中, 尽管石墨烯具有优异的导热、导电性能, 有利于质子转移而生成 $\mathrm{NH}_{3}$ 和 $\mathrm{HClO}_{4}{ }^{23}$ 但由于石墨 烯具有很大的比表面积, 在 AP 低温分解开始时会 吸附生成的 $\mathrm{NH}_{3}$ 和 $\mathrm{HClO}_{4}$, 从而延缓了二者进入气 相并发生氧化反应，因而造成 $\mathrm{AP} /$ 石墨烯复合材料 和 AP 与石墨烯的机械共混物的低温分解过程被抑 制. 只有达到较高温度时, $\mathrm{NH}_{3}$ 和 $\mathrm{HClO}_{4}$ 才能解吸并 在气相发生快速氧化还原反应. 与三种 $\mathrm{AP}$ 与石墨 烯的机械共混物相比, 在通过不同干燥方式制备的 $\mathrm{AP} /$ 石墨烯复合材料中, AP 是以纳米尺寸(或接近纳 米尺寸)与石墨烯复合的, 与石墨烯接触更加充分, 更有利于质子的转移和热量的传导, 因而石墨烯的 促进作用更加明显。

不同干燥方式制备的 AP/石墨烯复合材料和 AP 与石墨烯的机械共混物的表观分解热也有差异. 如表 1 所示, $\mathrm{AP}$ 的总表观分解热为 $621 \mathrm{~J} \cdot \mathrm{g}^{-1}$, 以不同 比例与石墨烯混合后, 三种 AP 与石墨烯的机械共 混物的总表观分解热分别达到 1987、1860 和 1786 $\mathrm{J} \cdot \mathrm{g}^{-1}$, 而自然干燥、冷冻干燥和超临界 $\mathrm{CO}_{2}$ 干燥制备 的 $\mathrm{AP} /$ 石墨烯复合材料的总表观分解热分别达到 $2090 、 2048$ 和 $2110 \mathrm{~J} \cdot \mathrm{g}^{-1}$. AP 与石墨烯复合后, 表观 分解热显著增加的主要原因是石墨烯与 AP热分解 产生的氧化性产物发生氧化反应, 产生了大量的
热, 致使总表观分解热增加. 不同干燥方式制备的 $\mathrm{AP} /$ 石墨烯复合材料的表观分解热的差异主要是由 干燥方式不同导致复合材料中 AP 的含量和粒径不 同造成的, 而前者直接导致了复合材料的氧平衡系 数的不同. 根据含能材料领域的氧平衡理论, 当含 能材料为零氧平衡时其分解放热量最大, 而当含能 材料为正氧平衡或负氧平衡时, 其分解放热量均要 下降. ${ }^{24,25}$ 根据元素分析结果, 通过不同干燥方式制 备的 $\mathrm{AP} /$ 石墨烯复合材料的氧平衡系数如表 1 所示. 自然干燥法和冷冻干燥法制备的 $\mathrm{AP} /$ 石墨烯复合材 料相比, 前者更接近零氧平衡, 因而放热量要更大 一些. 而就超临界 $\mathrm{CO}_{2}$ 干燥法制备的 $\mathrm{AP} /$ 石墨烯复 合材料而言, 因其中所含的 $\mathrm{AP}$ 处于纳米尺寸, AP与 石墨烯的接触更充分, 反应更加剧烈. 因此, 尽管其 氧平衡系数偏离零氧平衡最多, 但放热量仍然是三 种 $\mathrm{AP} /$ 石墨烯复合材料中最大的. 在三种 $\mathrm{AP}$ 与石墨 烯的机械共混物中, 石墨烯与 AP 的接触不如 AP/石 墨烯复合材料中充分, 因而氧化反应放热量要小一 些.

图 4 是纯 $\mathrm{AP}$ 、通过不同干燥方式制备的 $\mathrm{AP} /$ 石 墨烯复合材料和 AP 与石墨烯的机械共混物的 TG 曲线. 由图 4 可以看到, 纯 AP 的整个热分解过程出 现两个明显的质量损失阶段, 分别对应其低温分解 过程和高温分解过程. 通过自然干燥制备的 AP/石 墨烯复合材料和三种 AP 与石墨烯的机械共混物的 热分解过程也出现两个质量损失阶段, 但是它们的 低温分解过程的质量损失不明显，高温分解过程是 它们质量损失的主要阶段. 通过冷冻干燥和超临界 $\mathrm{CO}_{2}$ 干燥制备的 $\mathrm{AP} /$ 石墨烯复合材料的热分解过程 只出现一个质量损失阶段, 分别对应于二者的高温 分解过程, 这与 DSC 分析的结果是一致的.

\subsection{TG-FTIR 分析}

为了进一步研究 $\mathrm{AP} /$ 石墨烯复合材料的热分解 行为, 通过 TG-FTIR 联用技术分析了纯 AP 和通过

表 1 不同样品的AP含量、氧平衡系数和DSC 数据

Table 1 AP content, oxygen balance, and DSC data of different samples

\begin{tabular}{cccccc}
\hline Sample & $w_{\mathrm{AP}} / \%$ & Oxygen balence $/ \%$ & $T_{\mathrm{L}} /{ }^{\circ} \mathrm{C}$ & $T_{\mathrm{H}} /{ }^{\circ} \mathrm{C}$ & $\Delta H /\left(\mathrm{J} \cdot \mathrm{g}^{-1}\right)$ \\
\hline pure AP & 100.00 & 34.00 & 297.0 & 406.2 & 621 \\
mechanical mixtures of AP with graphene $\left(w_{\mathrm{AP}}=89.97 \%\right)$ & 89.97 & 3.79 & 304.0 & 370.2 & 1987 \\
mechanical mixtures of AP with graphene $\left(w_{\mathrm{AP}}=92.41 \%\right)$ & 92.41 & 11.15 & 305.8 & 364.1 & 1860 \\
mechanical mixtures of AP with graphene $\left(w_{\mathrm{AP}}=94.40 \%\right)$ & 94.40 & 17.14 & 297.3 & 339.3 & 1786 \\
AP/graphene with drying naturally in atmosphere & 89.97 & 3.79 & 293.3 & 336.5 & 2090 \\
AP/graphene with freeze drying & 92.41 & 11.15 & - & 322.0 & 2048 \\
AP/graphene with supercritical $\mathrm{CO}_{2}$ drying & 94.40 & 17.14 & - & 322.5 & 2110 \\
\hline
\end{tabular}

$w_{\mathrm{AP}}$ : mass fraction of AP, $T_{\mathrm{L}}$ : low-temperature exothermic peak temperature, $T_{\mathrm{H}}$ : high-temperature exothermic peak temperature, $\Delta H$ : total heat release 


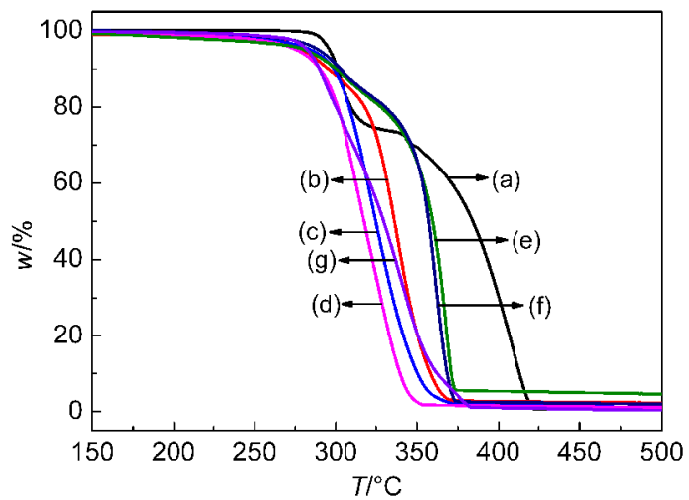

图 4 不同样品的 TG 曲线

Fig.4 TG curves of different samples

(a) pure $\mathrm{AP}$; (b) AP/graphene composites prepared by drying naturally in atmosphere; (c) AP/graphene composites prepared by freeze drying;

(d) $\mathrm{AP} /$ graphene composites prepared by supercritical $\mathrm{CO}_{2}$ drying;

(e) mechanical mixtures of AP with graphene $\left(w_{\mathrm{AP}}=89.97 \%\right)$;

(f) mechanical mixtures of AP with graphene $\left(w_{\mathrm{AP}}=92.41 \%\right)$;

(g) mechanical mixtures of AP with graphene ( $\left.w_{\mathrm{AP}}=94.40 \%\right)$

不同干燥方式制备的 AP/石墨烯复合材料的热分解 过程. 图 5 分别是纯 AP 和通过不同干燥方式制备的 $\mathrm{AP} /$ 石墨烯复合材料的热分解气相产物的三维 TG-FTIR 谱图. 从图 5 中可以看到, 纯 AP 的热分解 产物主要出现在 25-32 $\mathrm{min}$ 和 34-43 min, 并且其低 温分解产物和高温分解产物相似, 主要是 $\mathrm{HCl}$
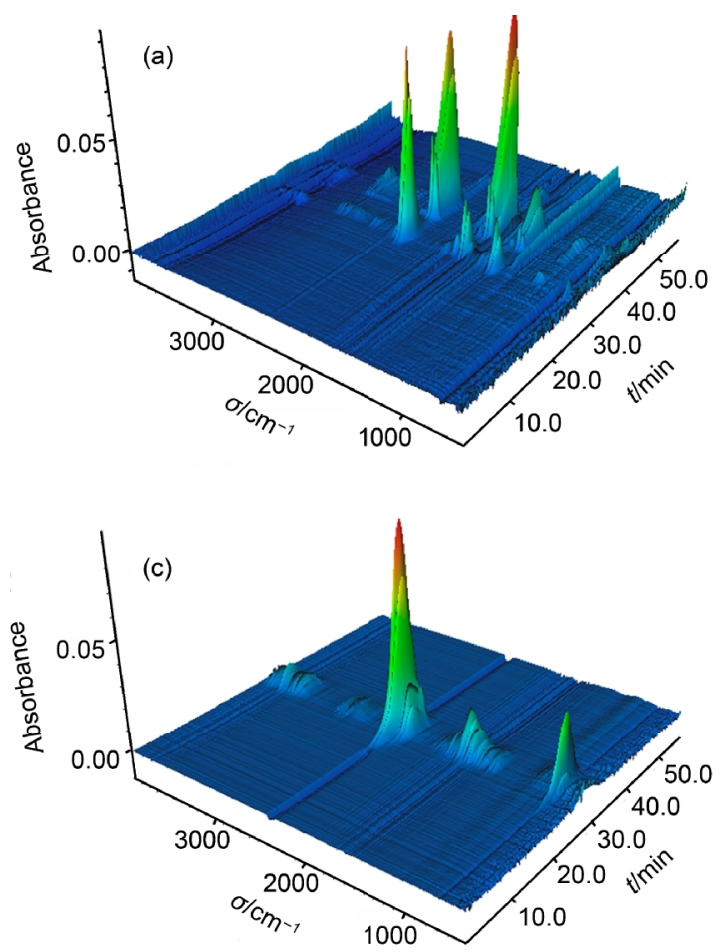

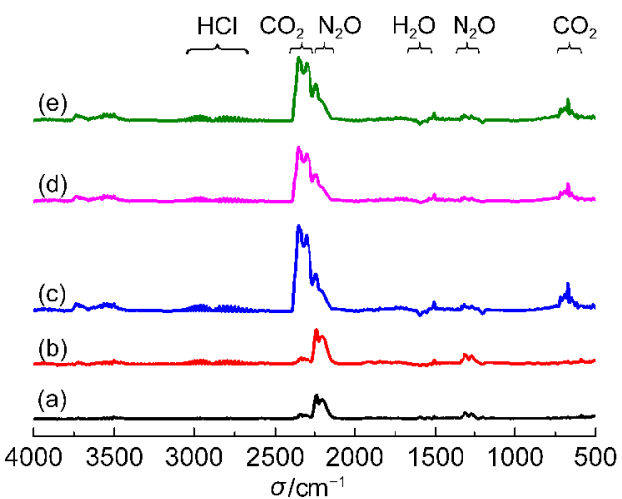

图6 不同样品在它们各自的低温或高温分解峰温时 热分解气相产物的 FTIR 谱图

Fig.6 FTIR spectra of gaseous products for different samples at the low/high-temperature exothermic peak temperature

(a) pure $\mathrm{AP}$ at the low-temperature exothermic peak temperature; (b) pure AP at the high-temperature exothermic peak temperature; $\mathrm{AP} /$ graphene composites prepared by (c) drying naturally in atmosphere, (d) freeze drying, and (e) supercritical $\mathrm{CO}_{2}$ drying at their respective high-temperature exothermic peak temperature

$\left(2640-3080 \mathrm{~cm}^{-1}\right), \mathrm{N}_{2} \mathrm{O}\left(1230-1350 \mathrm{~cm}^{-1}\right.$ 和 2120$\left.2260 \mathrm{~cm}^{-1}\right)$ 和少量 $\mathrm{H}_{2} \mathrm{O}\left(1540-1660 \mathrm{~cm}^{-1}\right)$. 对于通过 不同干燥方式制备的 $\mathrm{AP} /$ 石墨烯复合材料来说, 它 们的热分解产物主要出现在 22-36 min. 在它们的
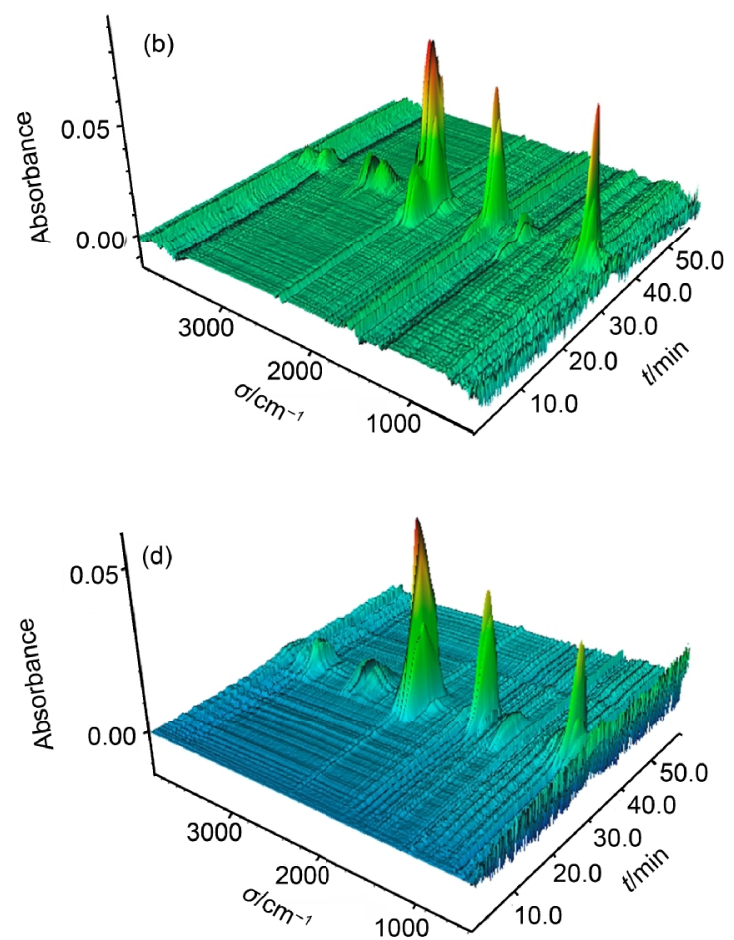

图 5 纯 $\mathrm{AP}(\mathrm{a})$ 、自然干燥(b)、冷冻干燥(c)和超临界 $\mathrm{CO}_{2}$ 干燥(d)制备的 $\mathrm{AP} /$ 石墨烯复合材料热分解气相产物的 三维 TG-FTIR 谱图

Fig.5 Three dimensional TG-FTIR spectra of gaseous products in thermal decomposition of pure AP (a), and AP/graphene composites prepared by drying naturally in atmosphere (b), freeze drying (c), and supercritical $\mathrm{CO}_{2}$ drying (d) 
三维 TG-FTIR 谱图中, 除了上述分解产物的特征峰 外, 均出现了 $\mathrm{CO}_{2}\left(610-730 \mathrm{~cm}^{-1}\right.$ 和 $\left.2270-2400 \mathrm{~cm}^{-1}\right)$ 的特征峰.

图 6 是纯 AP 在其低温分解峰温和高温分解峰 温时, 以及通过不同干燥方式制备的 $\mathrm{AP} /$ 石墨烯复 合材料在它们各自的高温分解峰温时热分解气相 产物的FTIR 谱图. 如图6 所示, 纯 AP 在其低温分解 峰温和高温分解峰温时热分解气相产物的 FTIR 谱 图上均出现了 $\mathrm{HCl} 、 \mathrm{~N}_{2} \mathrm{O}$ 和 $\mathrm{H}_{2} \mathrm{O}$ 的特征峰. 而通过不 同干燥方式制备的 $\mathrm{AP} /$ 石墨烯复合材料在它们各自 高温分解峰温时热分解气相产物的 FTIR 谱图上, $\mathrm{N}_{2} \mathrm{O}$ 和 $\mathrm{H}_{2} \mathrm{O}$ 的特征峰强度变弱, 并且出现了明显的 $\mathrm{CO}_{2}$ 特征峰, 这说明在热分解过程中石墨烯和 $\mathrm{AP}$ 分 解产生的氧化性产物发生了氧化反应, 生成了 $\mathrm{CO}_{2}$, 同时 $\mathrm{N}_{2} \mathrm{O}$ 的产生被抑制.

\section{4 结 论}

(1) 以氧化石墨烯为前驱体, 通过溶胶-凝胶法 制备了石墨烯水凝胶, 并通过溶液渗透过程将其与 $\mathrm{AP}$ 复合, 然后分别采用自然干燥、冷冻干燥和超临 界 $\mathrm{CO}_{2}$ 干燥三种干燥方式制备了 $\mathrm{AP} /$ 石墨烯复合材 料.

(2) 通过自然干燥制备的 AP/石墨烯复合材料 收缩严重, 表面有大量 AP 析出, 石墨烯团聚严重; 通过冷冻干燥制备的 $\mathrm{AP} /$ 石墨烯复合材料结构非常 疏松, 石墨烯骨架坍塌, 多孔结构不明显; 而通过超 临界 $\mathrm{CO}_{2}$ 干燥制备的 $\mathrm{AP} /$ 石墨烯复合材料基本能保 持与石墨烯气凝胶相似的外观和多孔结构.

(3) 元素分析和 X射线衍射分析结果表明, 通过 自然干燥、冷冻干燥和超临界 $\mathrm{CO}_{2}$ 干燥制备的 $\mathrm{AP} /$ 石墨烯复合材料中 AP 的质量分数分别为 $89.97 \%$ 、 $92.41 \%$ 和 $94.40 \%$, 其中通过超临界 $\mathrm{CO}_{2}$ 干燥制备的 $\mathrm{AP} /$ 石墨烯复合材料中 AP 的粒径尺寸为 $69 \mathrm{~nm}$.

(4) DSC 测试结果表明, 将 AP 和石墨烯以凝胶 形式复合后, 石墨烯对 AP 的热分解过程表现出明 显的促进作用. 与纯 $\mathrm{AP}$ 相比, 通过不同干燥方式制 备的 $\mathrm{AP} /$ 石墨烯复合材料的低温分解峰均大大削弱 或者完全消失, 高温分解峰温均显著降低. 其中, 通 过超临界 $\mathrm{CO}_{2}$ 干燥制备的 $\mathrm{AP} /$ 石墨烯复合材料中石 墨烯的促进作用最明显, 其高温分解峰温降低了 $83.7^{\circ} \mathrm{C}$, 表观分解热提高到 $2110 \mathrm{~J} \cdot \mathrm{g}^{-1}$. TG-FTIR 分 析结果表明, $\mathrm{AP} /$ 石墨烯复合材料的热分解过程中, $\mathrm{AP}$ 分解产生的氧化性产物与石墨烯发生了氧化反
应, 生成了 $\mathrm{CO}_{2}$, 并产生大量的热.

(5) 三种干燥方式相比, 通过超临界 $\mathrm{CO}_{2}$ 干燥制 备的 $\mathrm{AP} /$ 石墨烯复合材料中, $\mathrm{AP}$ 的含量最高, 且 $\mathrm{AP}$ 的粒径尺寸为纳米级. 与纯 $\mathrm{AP}$ 相比, 通过超临界 $\mathrm{CO}_{2}$ 干燥制备的 $\mathrm{AP} /$ 石墨烯复合材料的高温分解峰 明显提前, 表观分解热的提高也最为显著. 从综合 性能上来说, 三种干燥方式中超临界 $\mathrm{CO}_{2}$ 干燥是制 备 $\mathrm{AP} /$ 石墨烯复合材料最合适的干燥方式.

致谢：作者感谢北京理工大学材料学院张学同教授在石墨 烯水凝胶和气凝胶制备方面给予的热心指导.

\section{References}

(1) Zhou, L. M.; Liu, H. Y.; Li, F. S. Acta Phys. -Chim. Sin. 2006, $22(5), 627$. [周龙梅, 刘宏英, 李凤生. 物理化学学报, 2006, 22 (5), 627.] doi: 10.3866/PKU.WHXB20060521

(2) Liu, H. B.; Jiao, Q. Z.; Zhao, Y.; Li, H. S.; Sun, C. B.; Li, X. F.; Wu, H. Y. Mater. Lett. 2010, 64, 1698. doi: 10.1016/j.matlet. 2010.04 .061

(3) Liu, L. L.; Li, F. S.; Tan, L. H.; Min, L.; Yi, Y. Propellants Explos. Pyrotech. 2004, 29, 34.

(4) Kapoor, I. P. S.; Srivastava, P.; Singh, G. Propellants Explos. Pyrotech. 2009, 34, 351. doi: 10.1002/prep.v34:4

(5) Luo, X. L.; Han, Y. F.; Yang, D. S.; Chen, Y. S. Acta Phys. -Chim. Sin. 2012, 28 (2), 297. [罗小林, 韩银风, 杨德 锁, 陈亚苟. 物理化学学报, 2012, 28 (2), 297.] doi: 10.3866/ PKU.WHXB201112012

(6) Chandru, R. A.; Patra, S.; Oommen, C.; Munichandraiah, N.; Raghunandan, B. N. J. Mater. Chem. 2012, 22, 6536. doi: $10.1039 / \mathrm{c} 2 \mathrm{jm} 16169 \mathrm{a}$

(7) Li, N.; Cao, M. H.; Wu, Q. Y.; Hu, C. W. CrystEngComm 2012, 14, 428. doi: 10.1039/c1ce05858d

(8) Chaturvedi, S.; Dave, P. N. J. Exp. Nanosci. 2012, 7 (2), 205. doi: $10.1080 / 17458080.2010 .517571$

(9) Han, X.; Sun, Y. L.; Wang, T. F.; Lin, Z. K.; Li, S. F.; Zhao, F. Q.; Liu, Z. R.; Yi, J. H.; Ren, X. E. J. Therm. Anal. Calorim. 2008, 91, 551. doi: 10.1007/s10973-007-8290-6

(10) Reshmi, S.; Catherine, K. B.; Nair, C. P. R. Int. J. Nanotechnol. 2011, $8(10-12), 979$

(11) Compton, O. C.; Nguyen, S. T. Small 2010, 6, 711. doi: 10.1002/ smll.v6:6

(12) Geim, A. K.; Novoselov, K. S. Nat. Mater. 2007, 6, 183. doi: $10.1038 /$ nmat 1849

(13) Stoller, M. D.; Park, S. J.; Zhu, Y. W.; An, J. H.; Ruoff, R. S. Nano Lett. 2008, 8 (10), 3498. doi: 10.1021/n1802558y

(14) Du, X.; Skachko, I.; Barker, A.; Andrei, E. Y. Nat. Nanotechnol. 2008, 3 (8), 491. doi: 10.1038/nnano.2008.199

(15) Lee, C.; Wei, X. D.; Kysar, J. W.; Hone, J. Science 2008, 321, 385. doi: 10.1126/science. 1157996 
(16) Balandin, A. A.; Ghosh, S.; Bao, W. Z.; Calizo, I.; Teweldebrhan, D.; Miao, F.; Lau, C. N. Nano Lett. 2008, 8 (3), 902. doi: $10.1021 / \mathrm{nl} 0731872$

(17) Zhang, X. T.; Sui, Z. Y.; Xu, B.; Yue, S. F.; Luo, Y. J.; Zhan, W. C.; Liu, B. J. Mater. Chem. 2011, 21, 6494. doi: 10.1039/ cljm10239g

(18) Xu, Y. X.; Sheng, K. X.; Li, C.; Shi, G. Q. ACS Nano 2010, 4 (7), 4324. doi: 10.1021/nn101187z

(19) Chen, W. F.; Yan, L. F. Nanoscale 2011, 3, 3132. doi: 10.1039/ clnr10355e

(20) Hummers, W. S.; Offeman, R. E. J. Am. Chem. Soc. 1958, 80, 1339. doi: $10.1021 / \mathrm{ja} 01539 \mathrm{a} 017$

(21) Boldyrev, V. V. Thermochim. Acta 2006, 443, 1. doi: 10.1016/j. tca.2005.11.038

(22) Fan, X. Z.; Li, J. Z.; Fu, X. L.; Wang, H. Acta Chim. Sin. 2009, 67 (1), 39. [樊学忠, 李吉祯, 付小龙, 王 晗. 化学学报, 2009, 67 (1), 39.]

(23) Li, N.; Geng, Z. F.; Cao, M. H.; Ren, L.; Zhao, X. Y.; Liu, B.; Tian, Y.; Hu, C. W. Carbon 2013, 54, 124. doi: 10.1016/j. carbon.2012.11.009

(24) Lu, M.; Lü, C. X. Journal of Nanjing University of Science and Technology 2002, 26, 72. [陆 明, 吕春绪. 南京理工大学学 报, 2002, 26, 72.]

(25) Cooper, P. W. Explosives Engineering; Wiley-VCH: Albuquerque NM, 1996; pp 24-26. 